\title{
Generalized Tellegen Principle Used for Energy Method for Systems Modeling
}

\author{
Milan Stork \\ Dept. of Applied Electronics \\ and Telecommunications/RICE \\ stork@kae.zcu.cz
}

\author{
Daniel Mayer \\ Dept. of Theory of Electrical \\ Engineering \\ mayer@kte.zcu.cz
}

\author{
Josef Hrusak \\ Dept. of Theory of Electrical \\ and Telecommunications \\ hrusak@kae.zcu.cz
}

\begin{abstract}
This paper deals with dissipativity, stability, chaotic behavior and related structural properties of a relatively broad class of finite dimensional strictly causal systems. The class of nonlinear systems under consideration is described in the state-space representation form. System properties are investigated by a new approach based on a new abstract state energy concept, and on a proper generalization of the well known Tellegen's theorem as a form of the energy conservation principle. The resulting energy function is induced by the output signal power and determines both, the structure of a proper system representation as well as the corresponding system state space topology. The state minimality, as well as parameter minimality requirements plays a crucial role in the proposed method. Several examples are solved, and results of simulation are shown for illustration of fundamental ideas and basic attributes of the proposed method.
\end{abstract}

Keywords - chaos; energy; nonlinear; state space; Tellegen)

\section{INTRODUCTION}

In many real-world situations some natural concepts, such as causality principle and different forms of conservation laws, have generally been recognized as system properties of crucial practical importance. For example, the Tellegen's theorem is well known in the field of electrical engineering $[1,2]$. It is one of few general theoretical results that apply in non-linear and time-varying situations, too. In the paper a more general class of abstract strictly causal system representations is addressed. The proposed approach gets out from the hypothesis that any physically correct system representation must not be in contradiction not only with a set of measured data but also with a form of an energy conservation principle.

Thus, if a specific physical structure of the system under investigation would be explicitly known, then the concept of physical energy could serve as a fundamental tool for system analysis and synthesis.

\section{TELLEGEN'S THEOREM USED FOR SYSTEMS}

Tellegen's theorem is one of the most powerful theorems in network theory. Most of the energy distribution theorems and extremum principles in network theory can be derived from it. The Tellegen

Milan Stork's participation was supported by the European Regional Development Fund and the Ministry of Education, Youth and Sports of the Czech Republic under the Regional Innovation Centre for Electrical Engineering (RICE), project No. CZ.1.05/2.1.00/03.0094 and by the Internal Grant Agency of University of West Bohemia in Pilsen, the project SGS-2015-002 and GA15-22712S. theorem provides a useful tool to analyze complex network systems including electrical circuits, biological and metabolic networks, pipeline transport networks, and chemical process networks. The classical Tellegen's principle can be seen as main reasons for using it as a starting point of proposed method. The main aim of the contribution is to develop a new approach based on a generalized form of the classical Tellegen's principle as an abstract formulation of the energy conservation law, and to investigate some possibilities of its systematic use to solution of basic problems of nonlinear system theory [3 - 6]. Some connections of dissipativity, state and parameter minimality, instability and chaos with are investigated from this point of view. In order to explain essential features of the theorem [2], consider an arbitrarily connected electrical network with $n$ components and choose associated reference directions for branch voltages $v_{\mathrm{k}}$ and currents $i_{\mathrm{k}}$. Let Kirchhoff's laws be given by the following equations:

$$
A i(t)=0 ; \quad B v(t)=0
$$

where $A$ is a node incidence matrix, $B$ is a loop incidence matrix and $i(t), v(t)$ are defined as follows:

$$
\begin{aligned}
& i(t)=\left[i_{1}(t), i_{2}, \ldots, i_{n}(t)\right]^{T} \\
& v(t)=\left[v_{1}(t), v_{2}(t), \ldots v_{n}(t)\right]^{T}
\end{aligned}
$$

Let the vectors $i(t), v(t)$ be the elements of an Euclidean space $E_{n}$ and invoke the inner product:

$$
\langle i(t), v(t)\rangle=\sum_{k=1}^{b} i_{k}(t) v_{k}(t)
$$

Let $I$ be the set of all the vectors $i(t)$ and $V$ the set of all the vectors $v(t)$ satisfying the equations (1).

Theorem 1: (Tellegen's theorem) If $i(t) \in I$ and $v(t) \in V$ then it holds that:

$$
\langle i(t), v(t)\rangle=0
$$

It is worth noticing a close relation between physical correctness and Tellegen's theorem. It is also important to realize that the branch currents and voltages are chosen arbitrarily complied only with Kirchhoff's laws. It implies that different sets $\bar{I}, \bar{V}$ of the branch currents and voltages satisfying the laws can be selected and the relation:

$$
\langle\bar{i}(t), \bar{v}(t)\rangle=0, \bar{i}(t) \in \bar{I}, \bar{v}(t) \in \bar{V}
$$

still holds. 


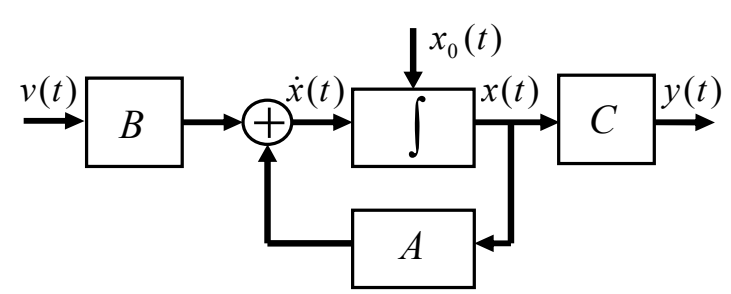

Figure 1. Block diagram of open loop system (without state or output feedback) with input $v(t)$, output $y(t)$, state $x(t)$, initial conditions $x_{0}(t)$ and matrices $A, B, C$

\section{GENERALIZED TELLEGEN'S PRINCIPLE}

Consider a class of state equivalent representations (see block diagram of Fig.1) described by equations (6) - (8) and structure [4] shown in Fig. 2.

$$
\begin{aligned}
& \frac{d x(t)}{d t}=A \cdot x(t)+B \cdot v(t) ; y(t)=C \cdot x(t)
\end{aligned}
$$

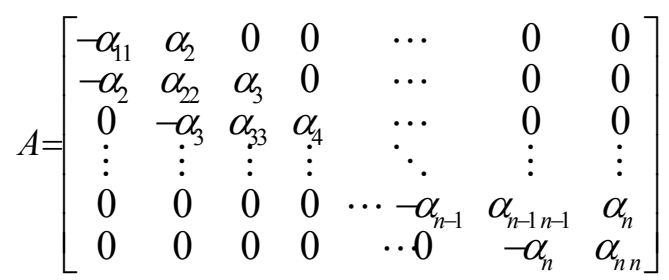

$$
\begin{aligned}
& B=\left[\begin{array}{llll}
\beta_{1} & \beta_{2} & \ldots & \beta_{n}
\end{array}\right]^{T} ; C=\left[\begin{array}{llll}
\gamma_{1} & \gamma_{2} & \ldots & \gamma_{n}
\end{array}\right]
\end{aligned}
$$

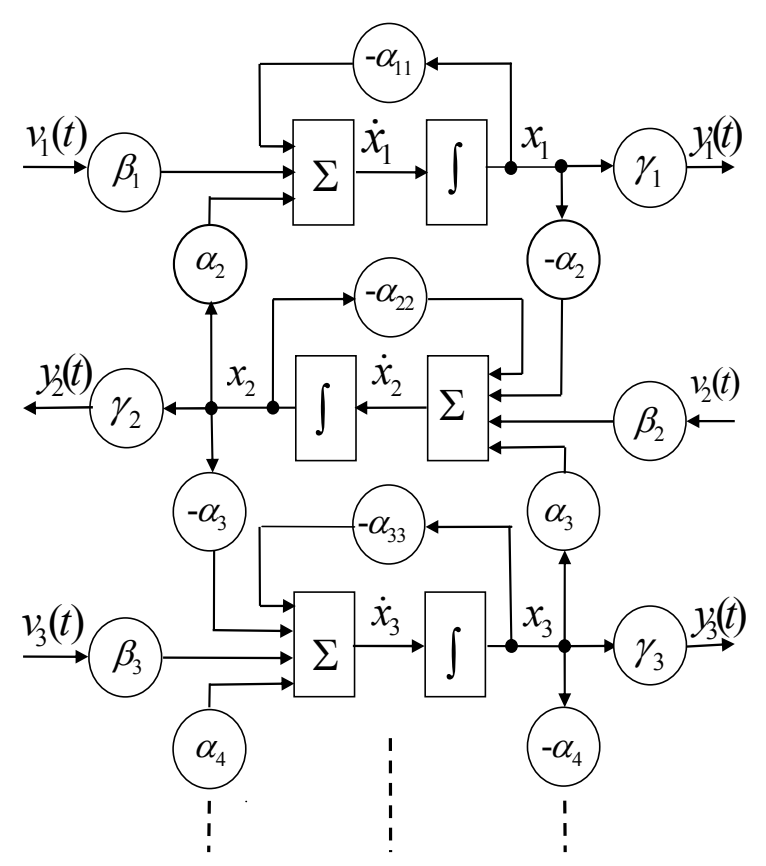

Figure 2. Physicaly correct structure for generalized Tellegen theorem $\left(\alpha_{\mathrm{i}}\right.$ should be function of $x_{\mathrm{i}}$ or time $)$

For the system described by previous equations and structure according Fig. 2 the generalized Tellegen theorem is given by

$$
\left\langle x^{T}(t), \frac{d x(t)}{d t}\right\rangle=0
$$

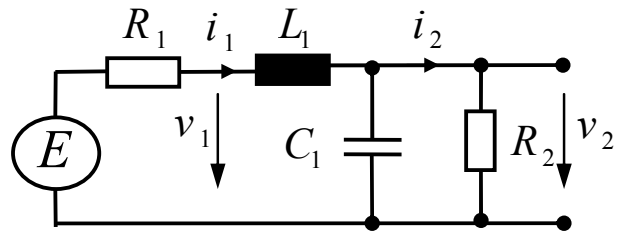

Figure 3. $R L C$ circuit used as linear example

\section{LINERAR SYSTEM EXAMPLE}

In this part the linear example is presented. Let us have a $2^{\text {nd }}$ order $R L C$ circuit shown in Fig. 3, which can be described by equations

$$
\begin{aligned}
& R_{1} i_{1}+L \frac{d i_{1}}{d t}+v_{2}=E \\
& C \frac{d v_{2}}{d t}+\frac{v_{2}}{R_{2}}=i_{1}
\end{aligned}
$$

State space equations are

$$
\left[\begin{array}{l}
\frac{d v_{2}}{d t} \\
\frac{d i_{1}}{d t}
\end{array}\right]=\left[\begin{array}{cc}
-\frac{1}{R_{2} C} & \frac{1}{C} \\
-\frac{1}{L} & -\frac{R_{1}}{L}
\end{array}\right] \cdot\left[\begin{array}{c}
v_{2} \\
i_{1}
\end{array}\right]+\left[\begin{array}{c}
0 \\
\frac{1}{L}
\end{array}\right] \cdot E
$$

or differential equation

$$
\begin{aligned}
& \frac{d^{2} i_{1}}{d t^{2}}+\left(\frac{R_{1}}{L}+\frac{1}{C R_{2}}\right) \frac{d i_{1}}{d t}+\left(\frac{1}{L C}+\frac{R_{1}}{L C R_{2}}\right) i_{1} \\
& =\frac{1}{L} \frac{d E}{d t}+\frac{E}{L C R_{2}}
\end{aligned}
$$

Tellegen equation for power is

$$
E \cdot i_{1}-\left(v_{R 1} i_{1}+v_{L} i_{L}+v_{C} i_{C}+v_{R 2} i_{R 2}\right)=0
$$

and after manipulation

$$
E \cdot i_{1}-\left(R_{1} i_{1}^{2}+L i_{1} \frac{d i_{1}}{d t}+C v_{C} \frac{d v_{C}}{d t}+\frac{v_{C}^{2}}{R_{2}}\right)=0
$$

From (18) can be derived also equation for energy

$$
\int_{0}^{t} E \cdot i_{1} d t-\left(\int_{0}^{t} R_{1} i_{1}^{2} d t+\frac{1}{2} L i_{1}^{2}+\frac{1}{2} C v_{C}^{2}+\int_{0}^{t} \frac{v_{C}^{2}}{R_{2}} d t\right)=0
$$

Suppose following values of circuit: $R_{1}=0.5[\Omega]$; $L=2.5[\mathrm{H}] ; C=0.1[\mathrm{~F}] ; \mathrm{R}_{2}=20[\Omega]$. Therefore eq. (11) is rewritten as

$$
\left[\begin{array}{l}
\frac{d v_{2}}{d t} \\
\frac{d i_{1}}{d t}
\end{array}\right]=\left[\begin{array}{cc}
-0.5 & 10 \\
-0.4 & -0.2
\end{array}\right] \cdot\left[\begin{array}{c}
v_{2} \\
i_{1}
\end{array}\right]+\left[\begin{array}{c}
0 \\
0.4
\end{array}\right] \cdot E
$$

State transformation leads to system described by

$$
\left[\begin{array}{c}
\frac{d x_{1}}{d t} \\
\frac{d x_{2}}{d t}
\end{array}\right]=\left[\begin{array}{cc}
-\frac{1}{R_{2} C} & \frac{1}{\sqrt{L C}} \\
-\frac{1}{\sqrt{L C}} & -\frac{R_{1}}{L}
\end{array}\right] \cdot\left[\begin{array}{l}
x_{1} \\
x_{2}
\end{array}\right]+\left[\begin{array}{c}
0 \\
\frac{1}{\sqrt{L C}}
\end{array}\right] \cdot v
$$




$$
\begin{aligned}
& \alpha_{11}=\frac{1}{R_{2} C} ; \alpha_{2}=\frac{1}{\sqrt{L C}} ; \alpha_{22}=-\frac{R_{1}}{L} ; \\
& \beta_{2}=\frac{1}{\sqrt{L C}} ; \gamma_{1}=1
\end{aligned}
$$

From generalized Tellegen equation (9) can be derived

$$
\begin{aligned}
& \left\langle x^{T}(t), \frac{d x(t)}{d t}\right\rangle=\underbrace{-\alpha_{11} x_{1}^{2}(t)-\alpha_{22} x_{2}^{2}(t)}_{P_{D}}+ \\
& \underbrace{\beta_{2} x_{2}(t) v(t)}_{P_{I}}=P_{D}(t)+P_{I}(t)=0
\end{aligned}
$$

where $P_{D}$ is dissipated power and $P_{I}$ is input power. Proof is given in next equations (20) - (22).

$$
\begin{aligned}
& x_{1}(t)=v_{C}(t) \sqrt{C} ; \quad x_{2}(t)=i_{1}(t) \sqrt{L} ; \quad v(t)=E \sqrt{C} \\
& P_{I}(t)=\beta_{2} x_{2}(t) v(t)=\frac{1}{\sqrt{L C}} i_{1}(t) \sqrt{L} \cdot E(t) \sqrt{C}= \\
& E(t) \cdot i_{1}(t) \\
& P_{D}(t)=-\alpha_{11} x_{1}^{2}-\alpha_{22} x_{2}^{2}= \\
& -\frac{1}{R_{2} C}\left(v_{C} \sqrt{C}\right)^{2}-\frac{R_{1}}{L}\left(i_{1} \sqrt{L}\right)^{2}=-\frac{v_{C}^{2}}{R_{2}}-R_{1} \cdot i_{1}^{2}
\end{aligned}
$$

The generalized Tellegen principle described by eq. (9) can be used also for system supplied only by initial condition as well for nonlinear system. It will show that type of system can be easily described by energy or power function. It is important to note that state space energy can be derived from power by

$$
V=\int_{0}^{t}\left\langle x^{T}, \dot{x}\right\rangle d t=\int_{0}^{t}\left(x_{1} \dot{x}_{1}+\ldots+x_{n} \dot{x}_{n}\right) d t=\sum_{i=1}^{n} x_{i}^{2}
$$

Time evolution of energy for $R L C$ circuit (Fig. 2), and time evolution of state space energy of abstract system described by eq. (19) are displayed in Fig. 4 response on initial condition and unit step. Booth curves are exactly same (therefore only one curve is visible in Fig. 4).
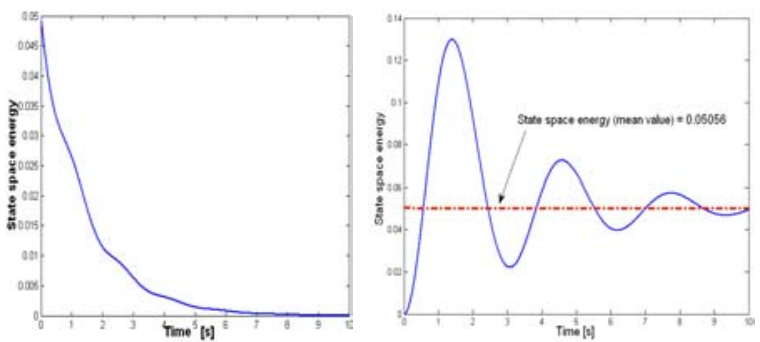

Figure 4. Left - Time evolution of energy in $R L C$ circuit (Fig. 3) and time evolution of state space energy, eq. (21) as response on initial conditions $v_{\mathrm{C}}(0)=1$ or $\boldsymbol{x}(0)=\left[x_{1}(0) x_{2}(0)\right]=\left[\begin{array}{ll}1 & 0\end{array}\right]^{\mathrm{T}}$. Booth curves are the same. Right - Time evolution of energy in $R L C$ circuit (Fig. 3) and time evolution of state space energy, eq. (21) as response on unit step. Mean value of energy $E_{\mathrm{C}}+E_{\mathrm{L}}=0.05056$. Booth curves are the same.

\section{NONLINEAR SYSTEM}

In this part the example of nonlinear (but nonchaotic) system is presented [7 - 8]. System is described by eq. (24) or block diagram (Fig. 6). For nonlinear function $f\left(x_{2}\right)$, two functions were used: $\left(x_{2}\right)^{2}$ and $a b s\left(x_{2}\right)$. Prescribed value is $w$ and $k$ is gain. The equivalent electronic system is shown in Fig. 8.

$$
\begin{aligned}
& \dot{x}_{1}=k \alpha_{11}\left(w-f\left(x_{2}\right)\right) x_{1}+\alpha_{2} x_{2} \\
& \dot{x}_{2}=-\alpha_{2} x_{1}-\alpha_{22} x_{2}
\end{aligned}
$$

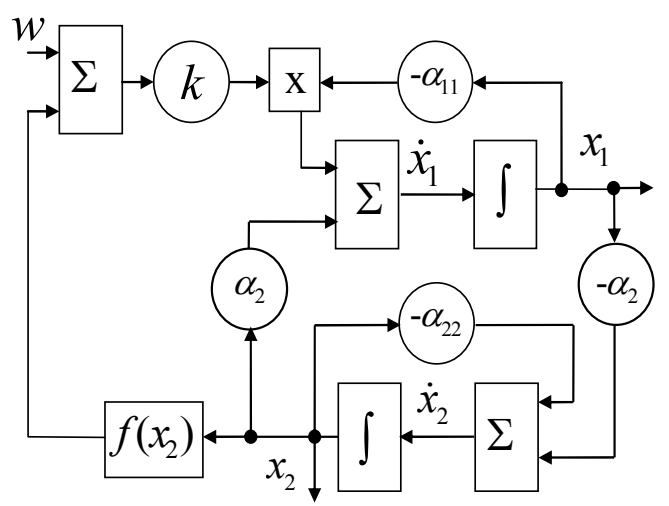

Figure 5. Block diagram of nonlinear system
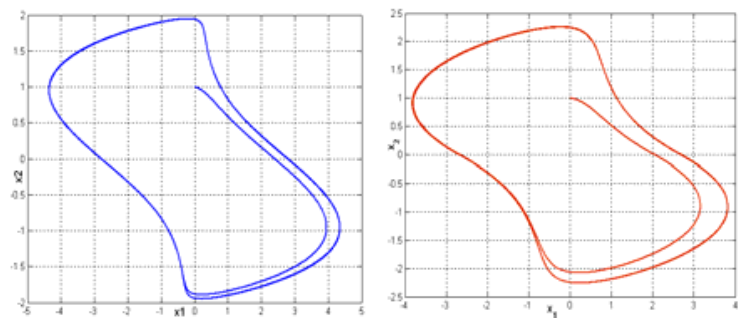

Figure 6. 2D phase portrait of nonlinear systems ( $x_{2}$ versus $\left.x_{1}\right)$. Left: $f\left(x_{2}\right)=x_{2}^{2} ; w=1 ; k=10$. Right: $f\left(x_{2}\right)=a b s\left(x_{2}\right) ; w=1 ; k=10$

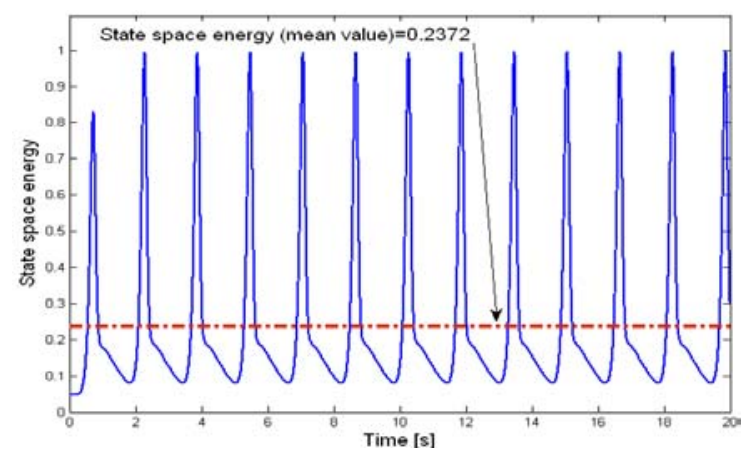

Figure 7. Time evolution of state space energy in nonlinear system for parameters: $f\left(x_{2}\right)=x_{2}^{2} ; w=1 ; k=10$;

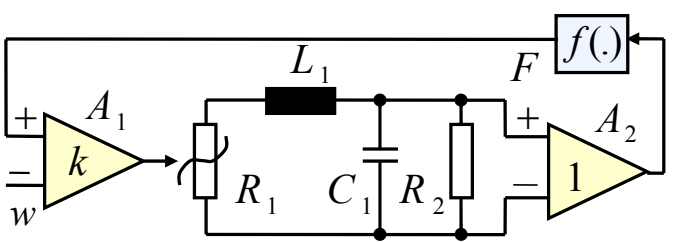

Figure 8. Equivalent nonlinear circuit. $A$ - instrumentation amplifiers with gain $k$ and $1 ; F$ - nonlinear function; $R_{1}$ - controlled resistor; $L_{1}, C_{1}, R_{2}$ - passive parts.

\section{CHAOTIC SySTEM}

The chaotic system is given by eq. (25) and structure see Fig. 9, where $w$ is prescribed value and $k_{2}$ 
and $k_{3}$ are gains [9 - 10]. Simulation results for $w=1$; $\alpha_{2}=1 ; \quad \alpha_{3}=0.89 ; \quad \alpha_{33}=0.1 ; \quad k_{2}=0.1 ; \quad k_{3}=3$ and initial condition [ $\left[\begin{array}{lll}0 & 0.1 & 0\end{array}\right]$ are shown in Fig, 10 - 12.

$$
\begin{aligned}
& \dot{x}_{1}=\left(w-k_{2} x_{2}^{2}-k_{3} x_{3}^{2}\right) x_{1}-\alpha_{2} x_{2} \\
& \dot{x}_{2}=\alpha_{2} x_{1}-\alpha_{3} x_{3} \\
& \dot{x}_{3}=\alpha_{3} x_{2}-\alpha_{33} x_{3}
\end{aligned}
$$

Chaotic behavior can be controlled by means of values $k_{2}$ and $k_{3}$.

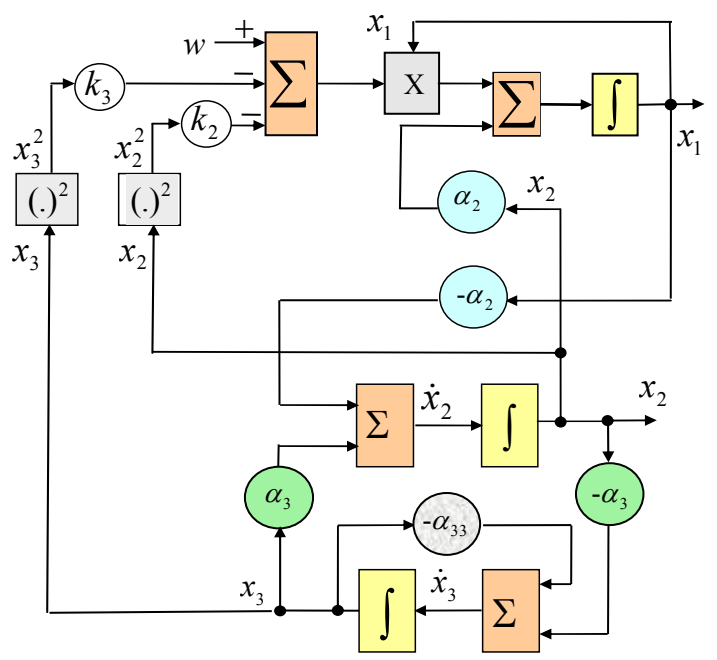

Figure 9. Structure of chaotic system
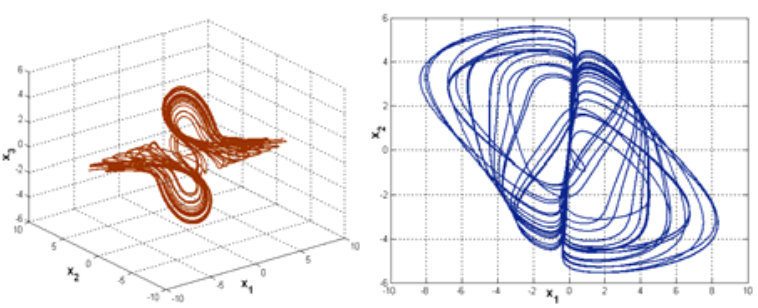

Figure 10. 3D phase portrait of chaotic system (left) and 2D phase portrait, $x_{2}$ versus $x_{1}$ (right)

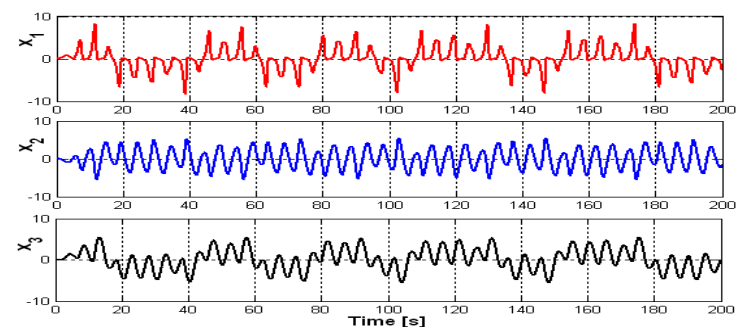

Figure 11. Time evolution of signals of chaotic system

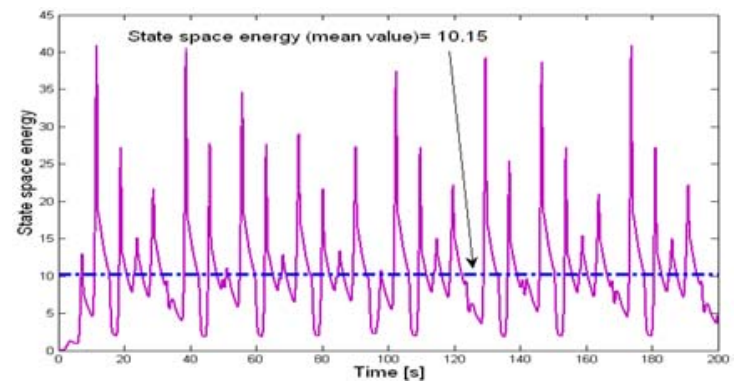

Figure 12. Time evolution of state space energy of chaotic system

\section{CONCLUSION}

In this paper the generalized Tellegen principle was used for power or energy description of different types of physical systems. It was derived, that abstract state space energy based on Tellegen principle can be used for linear, nonlinear or chaotic system. Most important is, that time evolution of energy can be used for classification of systems - for nonlinear system is periodic around the mean value, for chaotic system is non-periodic around mean value.

\section{REFERENCES}

[1] R. P. Ramachandran and V. Ramachandran, "Tellegen's Theorem Applied to Mechanical, Fluid and Thermal Systems," In: Proceedings of the 2001 American Society for Engineering Education Annual Conference \& Exposition, 2001.

[2] A. Bostan, G. Lecerf, E. Schost, "Tellegen's Principle into Practice," ISSAC'03, Philadelphia, Pennsylvania, USA, August 3-6,2003.

[3] J. Hrusak, D. Mayer and M. Stork, "On System Structure Reconstruction Problem And Tellegen-Like Relation," In: Proc. of $8^{\text {th }}$ World Multiconf.,SCI, Vol. VIII, Florida, USA, 2004, pp. 373-378.

[4] J. Hrusak, D. Mayer and M. Stork, "New approach to nonlinear instability and chaos based on generalized Tellegen's principle," In: WMSCI 2006. Orlando, Florida, International Institute of Informatics and Systemics, 2006, pp. 199-206.

[5] J. Hrusak, M. Stork, D. Mayer, "Generalized Tellegen`s Principle and state space energy based causal systems description," In: Advances in Energy Research: Distributed Generations Systems Integrating Renewable Energy Resources, Part I, Basic theory and advanced approaches, Chapter 4., NOVA Science Publ., USA, 2011, pp. 95-139.

[6] H. K. Khalil, "Nonlinear Systems, " Prentice Hall, 1996.

[7] M. Stork, J. Hrusak, D. Mayer, "Nonlinearly Coupled Oscillators and State Space Energy Approach", 14th WSEAS International Conference on Systems, Corfu Island, Greece, July 22-24, 2010, ISSN: 1792-4235, ISBN: 978960-474-199-1, pp. 171-175

[8] J. Hrusak, M. Stork, D. Mayer, "On State Space Energy Controlled Systems with Quantum Chaotic-Like Behavior", WSEAS/NAUN International Conferences, Corfu Island, Greece, July 14-17, 2011, pp. 165-170

[9] M. Stork, J. Hrusak, D. Mayer: "Energy based State Space Approach of Nonlinear Systems Simulation and Construction by means of electronic circuits", Recent Researches in Circuits and Systems, Proceedings of the 16th WSEAS International Conference on Systems ISBN: 978-161804-108-1, 2012, pp.78-84.

[10] M. Stork, J. Hrusak, D. Mayer, "On Synthesis Realization and Simulation Non-degenerate Dissipative Structures", Recent Advances in Telecommunications and Circuit Design, WSEAS/NAUN International Conferences Rhodes Island, Greece, July 16-19, 2013, pp. 49-56 